\title{
The Mitochondrial Pyruvate Carrier and Metabolic Regulation
}

\author{
Bor Luen Tang \\ Department of Biochemistry, Yong Loo Lin School of Medicine, National University of Singapore, Singapore \\ Email: bchtbl@nus.edu.sg
}

Received 4 October 2014; revised 3 November 2014; accepted 2 December 2014

Copyright (C) 2014 by author and Scientific Research Publishing Inc.

This work is licensed under the Creative Commons Attribution International License (CC BY). http://creativecommons.org/licenses/by/4.0/

c) (i) Open Access

\begin{abstract}
Pyruvate is a key intermediate at the branchpoint of anaerobic and aerobic energy metabolism. Its transport into the mitochondrial matrix is necessary prior to its decarboxylation into acetyl-CoA, which feeds the reducing equivalent-generating tricarboxylic acid (TCA) cycle. Although the existence of specific carrier transport of cytosolic pyruvate into the mitochondria has been inferred from a myriad of studies, the identities of the mitochondrial pyruvate carrier (MPC) were only confirmed very recently. Identification of the MPC facilitated several other recent advances. These include the finding of MPC's inhibition by the insulin-sensitizing drug family thiazolidinediones, how cells respond flexibly to a reduction in MPC functionality, as well as insights into how changes in MPC levels affect oncogenic potential of cancer cells. These new findings, discussed here in this brief review, have important implications in therapeutic approaches towards metabolic disorders and cancer.
\end{abstract}

\section{Keywords}

Cancer, Energy Metabolism, Mitochodrial Pyruvate Carrier (MPC), Pyruvate, Warburg Effect

\section{Introduction}

The 3-carbon monocarboxylate pyruvate is a product of cytosolic glycolysis, and is a key meeting point of the principal metabolic pathways of sugars, fatty acids and amino acids. In anaerobic glycolysis, pyruvate could be fermented to lactate in the cytosol by lactate dehydrogenase (LDH). In aerobic respiration, pyruvate needs to enter the mitochondria and be decarboxylated into the 2-carbon acetyl-CoA that feeds into the TCA cycle in the mitochondrial matrix. Pyruvate could be converted back to glucose by the process of gluconeogenesis and deaminated to form the amino acid alanine. In the mitochondria, it could also be carboxylated into the 4-carbon oxaloacetate (OAA), which could enter the TCA cycle or participate in anabolic pathways of glucose, amino 
acid and fatty acid synthesis (Figure 1).

While the need for cytosolic pyruvate to be transported into the mitochondrial matrixin aerobic respiration is basic textbook knowledge, how exactly pyruvate is transported was somewhat controversial. The outer mitochondrial membrane (OMM) is rather permeable to small molecules, but pyruvate in its ionized form is unlikely to negotiate the inner mitochondrial membrane (IMM) by passive diffusion. Although there was some early evidence for a significant degree of pyruvate free diffusion across artificial membranes, subsequent studies using purified mitochondria demonstrated saturation kinetics [1], thus suggesting the existence of a specific carrier(s). This notion is supported by the discovery of an inhibitor, the cinnamate $\alpha$-cyano-4-hydroxycinnamate (CHC) [2], which inhibited pyruvate oxidation in intact but not disrupted mitochondria [3]. The cinnamate derivativealphacyano-beta-(1-phenylindol-3-yl)acrylate] (UK5099) was shown to diminish labelling by [ $\left.{ }^{3} \mathrm{H}\right] \mathrm{N}$-phenylmaleimide (a thiol-blocking agent which inhibits pyruvate transport) of a $15 \mathrm{kDa}$ protein in heart and liver mitochondria [4]. The molecular size of this unidentified protein actually coincided well with those MPC components eventually found (which are small proteins of $14-16 \mathrm{kDa}$ in size) [5]. The exact molecular identity of the MPC has, however, remained elusive for some time. Its recent identification shall be described in more detail below.

As a key metabolic node, enzymes directly involved in pyruvate metabolism are tightly regulated [6] [7]. Likewise, pyruvate influx into the mitochondria is also likely regulated by multiple inputs from connecting pathways, and these regulatory mechanisms could be defective in disease conditions. Importantly, dysregulation of pyruvate mitochondrial influx could tip the balance between aerobic respiration and anaerobic glycolysis. A prominent example of such an imbalance is the Warburg effect [8]. As was observed and documented by Nobelist Otto Warburg in 1927 [9], tumor cells preferentially produce energy by heightened glycolysis and pyruvatelactate fermentation rather than oxidation of pyruvate in mitochondria. Although the effect could be exaggerated

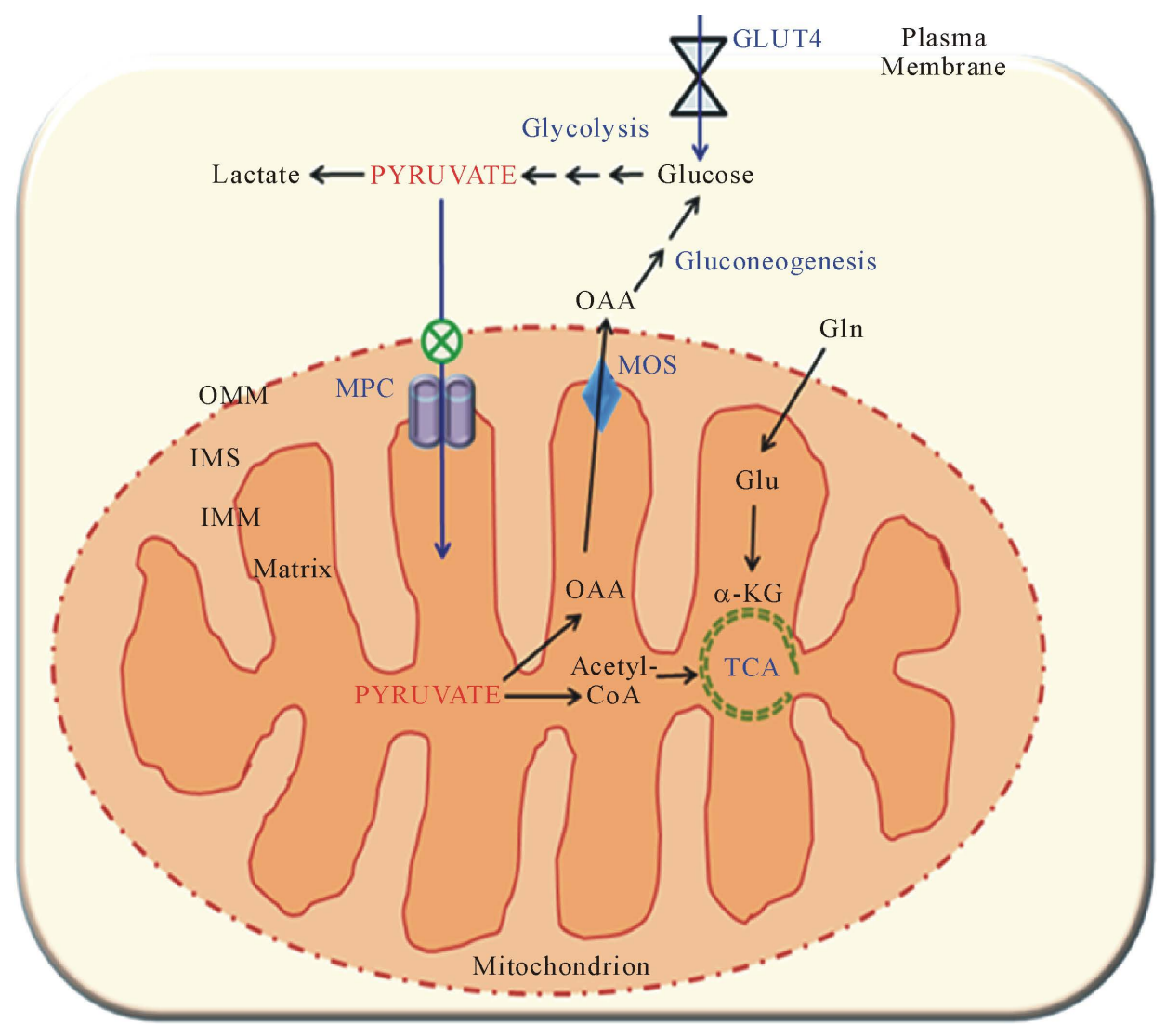

Figure 1. Schematic diagram of a mitochondrion illustrating the cellular components associated with pyruvate transport and metabolism. MPC-mitochondrial pyruvate carrier; MOS-MalateOAA shuttle; OMM - outer mitochondrial membrane; IMS — intermembrane space; IMM-inner mitochondrial membrane; OAA—oxaloacetate; $\alpha$-KG— $\alpha$-ketoglutarate; TCA—tricarboxylic acid cycle. 
by an increased intumor-specific pyruvate kinase isozyme M2 (PKM2) [10] and LDH, or a general mitochondrial dysfunction in cancer cells, defects in pyruvate influx is also a possibility. Pyruvate uptake by isolated mitochondria is a relatively slow process and evidence suggests that mitochondrial pyruvate transport might be rate limiting for mitochondrial pyruvate oxidation [11] [12].

With the molecular identification of MPC [13] [14], investigations into its role in metabolic regulation in normal can cancer cells are now feasible. In this brief review, I shall provide an update of some recent findings associated with the MPC.

\section{Identification of the Mitochondrial Pyruvate Carrier (MPC) Complex}

Identification of $\mathrm{CHC}$ as an inhibitor of pyruvate transport activity facilitated early attempts at partial purification and cell-free functional constitution of MPC [15]-[17]. Attempts were also made with affinity chromatography on immobilized CHC [18]. These attempts did not lead to any definitive identification of polypeptides that correspond to a functional MPC. A possible breakthrough came in 2003 when Halestrap's laboratory identified a possible MPC candidate in the yeast $S$. cerevisiae by a genetics approach. The authors measured UK5099-sensitive pyruvate uptake into mitochondria from 18 mitochondrial carrier family (MCF) deletion mutants. Only one mutant, YIL006W, exhibited no inhibitor-sensitive pyruvate transport, and the gene encodes a MCF family protein with likely mammalian homologues [19]. However, YIL006W was later shown by cloning and liposomal functional reconstruction assays to be one of two isoforms of the yeast $\mathrm{NAD}^{+}$transporters (Ndt1p) [20]. The highly anticipated molecular identification of MPC had to wait for almost another 10 years.

Rutter's laboratory was examining groups of mitochondrial proteins that are evolutionarily conserved, and noted the homologous YGL080W, YHR162W, and YGR243W genes which encode yeast Mpc1, Mpc2 and Mpc3, respectively [13]. The authors localized the gene products of these, which encode small polypeptides of 14 - 16 $\mathrm{kDa}$, to the mitochondrial inner membrane. $M p c 1$ and $M p c 2$ were found to form a multimeric complex of $\sim 150$ $\mathrm{kD}$. $m p c 1 \Delta$ and $m p c 2 \Delta$ cells (but not $m p c 3 \Delta$ ) displayed mild growth defects on nonfermentable carbon sources, and Drosophila or human MPC1 orthologs, could both rescue the mpc1 $1 \Delta$ phenotype. The Drosophila dMPC1 mutants have defective carbohydrate metabolism as they are sensitive to a diet consisting of only carbohydrates. Metabolomic analyses revealed that dMPC1 mutants on a sugar diet had high levels of pyruvate but a significant depletion of TCA cycle intermediates. A corresponding elevation/reduction profile of amino acids and other intermediates that could be derived from either cytosolic pyruvate or mitochondrial acetyl-CoA was also observed. Clear functional evidence that these genes encode MPC components came from a combination of biochemical and genetic experiments. Mitochondria of yeast $m p c 1 \Delta$ mutant exhibited no ${ }^{14} \mathrm{C}$-pyruvate uptake, a defect that could be effectively rescued by transgenic MPC1 in a MPC inhibitor UK5099-sensitive manner. The authors have also identified a yeast MPC1 mutation, Asp118 $\rightarrow$ Gly, which confers resistance to the MPC inhibitor UK5099. A very clinically relevant and significant finding of the Rutter's group is the identification of $B R P 44 L$ (human MCP1) mutations in families with impaired basal and uncoupling agent FCCP-stimulated pyruvate oxidation. Transgenic expression of wild type human MPC1 in cells derived from patients rescued the pyruvate oxidation defect, while mutant forms of human MPC1s have diminished or no rescue effect of the yeast deletion.

The Martinou group discovered MPC components while investigating defects in the synthesis of lipoic acid, a co-factor of several multi-subunit dehydrogenase complexes in the mitochondrial matrix [14]. The authors have previously identified Brp44L (human MPC1) in a proteomics analysis [21] and noted its homology with the yeast $Y G L 080 W, Y H R 162 W$, and $Y G R 243 W$ genes. They also found that the $m p c 1 \Delta$ and $m p c 2 \Delta m p c 3 \Delta$ deletion mutants grew more slowly in amino acid-free medium, a phenotype that was relieved by addition of the amino acids valine or leucine, but not other amino acids. ${ }^{14} \mathrm{C}$ tracer analysis showed that $m p c 1 \Delta$ cells have drastically reduced ${ }^{14} \mathrm{CO}_{2}$ release that corresponded with decreased dehydrogenase activities and their lipoylation, and lipoic acid production. The authors traced these defects to an upstream event of acetyl-CoA production and mitochondrial uptake of ${ }^{14} \mathrm{C}$ pyruvate, and like the Rutter group, found that mammalian (mouse) MPC1 could rescue the yeast defects. A key additional functional test performed by the Martinou group was the expression of mMPC1 and mMPC2, alone and in combination, in the bacteria Lactococcus lactis, a naïve system. Expression of both MPC proteins resulted in pyruvate uptake that was sensitive to UK5099, and with similar properties to mitochondrial pyruvate transport.

The genetic conservation and compelling biochemical and functional evidence for the MPCs discovered in the works described above provided strong evidence that the real MPC has now been identified. The polypeptides 
$M p c 1$ and $M p c 2$ form functional multimeric complexes at the IMM to mediate pyruvate translocation. It would appear that $\mathrm{Mpc3}$, which is highly homologous to $\mathrm{Mpc2}$, may have functional redundancy with the latter. Some caveats to this notion have been pointed out by Halestrap [22], and pyruvate transport by the purified protein(s) functionally reconstitution of into liposomes have not yet been demonstrated. We now await the structural analysis of these proteins in anticipation of the functional insights that shall be obtained. Interestingly, another recent report implicated an Arabidopsis thaliana gene, NRGA1, which is homologous to MPC2 and when coexpressed with AtMPC1, complemented the yeast $m p c 2 \Delta / m p c 3 \Delta$ mutation [23]. NRGA1 negatively regulates abscisic acid-induced signaling in Arabdopsis guard cells in response to drought, but how this is connected to pyruvate transport is unclear at the moment.

\section{Aspects of Metabolic Regulation Unveiled by MPC Inhibition}

Several recent studies have been aided by the molecular identification of MPC. Murphy and colleagues found that the anti-diabetic drug family of thiazolidinediones (TZDs), better known as peroxisome proliferator-activated receptor gamma (PPAR $\gamma$ ) inhibitors [24], also inhibits MPC at clinically relevant concentrations [25]. The authors showed that low dosages of TZD acted like UK5099 and specifically inhibited pyruvate and not glutamate of succinate oxidation in several cell types. The IMM-permeable methyl pyruvate rescued both the TZD and UK5099 inhibition, as it did the respiratory defects in cells with MPC1 or MPC2 silenced by lentiviralshRNA. Importantly, MPC inhibition by TZD underlies its stimulation of glucose uptake, degrees of which in myotubes and myocytes were directly proportional to the level of respiratory inhibition by TZDs or UK5099. TZD activation of the energy status-sensing, catabolism driver AMP-activated protein kinase (AMPK) [26], is also mimicked by UK5099. In another report, MCP1 and MCP2 were identified as mitochondrial proteins that could be chemically crosslinked to TZD [27] in a manner that could be blocked by UK5099. These authors also showed that TZDs altered the incorporation of ${ }^{13} \mathrm{C}$-labeled carbon from glucose into acetyl CoA. These findings thus connect mitochondrial pyruvate uptake to acute responses in glucose sensing and uptake, which could be therapeutically useful.

Another recent finding further suggests that MPC activity affects glucose sensing and insulin sensitivity. In mice, loss of $M p c 2$ is embryonically lethal, but Vigueira et al. generated a viable MPC2 hypomorphic mouse line harboring an N-terminally truncated protein and exhibiting reduced capacity for mitochondrial pyruvate oxidation [28]. These mice have elevated blood glucose and lactate when subjected to an intraperitoneal pyruvate bolus. The mice are insulin-sensitive, but had reduced plasma insulin. Glucose intolerance in this strain was attributed to impaired glucose-stimulated pancreatic insulin secretion, which could be corrected by sulfonylurea treatment. This rather specific insulin secretion defect in a MPC2 hypomorph is interesting, and attested again to a connection between mitochondrial pyruvate uptake and glucose sensing.

What exactly would the consequences of MPC inhibition be on the core metabolic pathways such as the TCA cycle? A recent study by Vacanti et al. using ${ }^{13} \mathrm{C}$ metabolic flux analysis of cells after genetic or pharmacological ablation of MPC activity revealed a surprising degree of cellular metabolic flexibility and adaptation [29]. In MPC-deficient cells, both glucose and pyruvate oxidation were suppressed. However, the authors found that cell growth, oxygen consumption, and the TCA cycle functionality were surprisingly maintained by enhanced oxidative glutaminolysis. Also, MPC silencing increased fatty acid $\beta$-oxidation and branched-chain amino acid oxidation. This response is therefore unlike those observed during inhibition of the electron transport chain complex I or PDH, and cells could apparently reprogram to adapt to a reduction in mitochondrial pyruvate transport by channeling in products of amino acids and fatty acid metabolism to feed the TCA cycle.

The findings of Vacanti et al. are mirrored by another report. Yang et al. have previously found that glucose deprivation in c-Myc transformed cancer cells prompted acetyl-CoA generation via glutamine, which isconverted into glutamate by glutaminase and subsequently $\alpha$-ketoglutarate by elevated glutamate dehydrogenase (GDH) activity [30]. With tracer experiments, the authors now found that glucose and pyruvate transport into mitochondria suppresses GDH and acetyl-CoA formation from glutamine [31]. Impairment of pyruvate transport into mitochondria by UK5099 inhibition of MPC conversely induces glutamine-dependent acetyl-CoA formation. While UK5099 and glutaminase or GDH inhibitors only moderately suppressed cell proliferation and did not cause significant cell death, a combination UK5099 with inhibition of either glutaminase or GDH synergistically increased growth suppression and cell death. Interestingly, a combination of MPC and GDH inhibitors also impaired tumor growth in mouse xenografts. Other than uncovering aspects of metabolic flexibility, these 
results also suggest that pyruvate transport may be considered as a potential target in cancer therapeutics.

\section{MPC, Mitochondrial Pyruvate Transport and Cancer}

Cancer cells have profound metabolic alterations compared to noncancerous cells [32]. One prominent feature, termed the Warburg effect [8], is highlighted by the fact that many cancer cells tend to generate lactate from pyruvate and have reduced aerobic oxidation, even under normoxic and aerobic conditions. This has been attributed to mitochondrial damage and impaired aerobic respiration. However, many cancer cells with an intact TCA cycle nonetheless exhibit the Warburg effect. Alternative explanations for the effect include decrease pyruvate production by PKM2, upregulated LDH and increase expression of PDH kinase PDK1. Rutter's group now showed that impaired mitochondrial transport due to MPC1 deletion or diminished expression could also explain the Warburg effect [33]. The authors found that while the gene locus of MPC2 is not frequently loss or altered in cancer, the MPC1 locus is within the most frequently deleted region across all cancer samples investigated. MPC1 expression is also reduced in all cancer types examined, with its reduced expression correlating with poor disease prognosis. Re-expressing of MPC1 and MPC2 in cells increased mitochondrial pyruvate oxidation, and interestingly impaired anchorage-independent growth and xenograft growth of these cells. The cancers cells therefore appeared to lose oncogenic potential, but did not suffer impaired health or viability.

How exactly does expression of MPC reverse the cancer phenotype? The authors noted that the enzyme aldehyde dehydrogenase (ALDH), a cancer stem cell marker [34] [35], was significantly decreased in the MPC-expressing tumors. Further examination revealed other stem cell markers such as LGR5 [36], LIN28A [37] and NANOG [38] were decreased in cells upon MPC expression. The mitochondrial pyruvate transport, or indeed the carrier itself, could have profound effects on the oncogenic expression profile of cancer cells beyond the superficially perceived alteration in metabolic profile.

\section{Concluding Remarks}

In the paragraphs above, recent advances in the molecular cloning and identification of the mitochondrial transport carrier were outlined, and some recent intriguing findings that have been facilitated by the discovery of MPC were discussed. Much remains to be learned about the MPC-mediated pyruvate transport process itself, such as the mechanism of transport and its regulation. Manipulations of MPC-dependent pyruvate transport into the mitochondria through MPCs have already revealed some surprising findings pertaining to metabolic flexibility of cells, and we could expect more revelations along this line in the near future. That MPC levels or function affect expression profiles of cancer stem cell markers is unexpected and exciting. We could look forward to the resolution of the underlying mechanism, and anticipate how this might become applicable in terms of cancer therapeutics.

\section{Acknowledgements}

The author declares no conflict of interest.

\section{References}

[1] Papa, S., Francavilla, A., Paradies, G. and Meduri, B. (1971) The Transport of Pyruvate in Rat Liver Mitochondria. FEBS Letters, 12, 285-288. http://dx.doi.org/10.1016/0014-5793(71)80200-4

[2] Halestrap, A.P. and Denton, R.M. (1974) Specific Inhibition of Pyruvate Transport in Rat Liver Mitochondria and Human Erythrocytes by alpha-Cyano-4-hydroxycinnamate. Biochemical Journal, 138, 313-316.

[3] Halestrap, A.P. and Denton, R.M. (1975) The Specificity and Metabolic Implications of the Inhibition of Pyruvate Transport in Isolated Mitochondria and Intact Tissue Preparations by alpha-Cyano-4-hydroxycinnamate and Related Compounds. Biochemical Journal, 148, 97-106.

[4] Thomas, A.P. and Halestrap, A.P. (1981) Identification of the Protein Responsible for Pyruvate Transport into Rat Liver and Heart Mitochondria by Specific Labelling with [3H]N-Phenylmaleimide. Biochemical Journal, 196, 471-479.

[5] Schell, J.C. and Rutter, J. (2013) The Long and Winding Road to the Mitochondrial Pyruvate Carrier. Cancer \& Metabolism, 1, 6.

[6] Gray, L.R., Tompkins, S.C. and Taylor, E.B. (2014) Regulation of Pyruvate Metabolism and Human Disease. Cellular 
and Molecular Life Sciences, 71, 2577-2604. http://dx.doi.org/10.1007/s00018-013-1539-2

[7] Jeoung, N.H., Harris, C.R. and Harris, R.A. (2014) Regulation of Pyruvate Metabolism in Metabolic-Related Diseases. Reviews in Endocrine \& Metabolic Disorders, 15, 99-110. http://dx.doi.org/10.1007/s11154-013-9284-2

[8] Palsson-McDermott, E.M. and O’Neill, L.A.J. (2013) The Warburg Effect Then and Now: From Cancer to Inflammatory Diseases. Bioessays, 35, 965-973. http://dx.doi.org/10.1002/bies.201300084

[9] Warburg, O., Wind, F. and Negelein, E. (1927) The Metabolism of Tumors in the Body. The Journal of General Physiology, 8, 519-530. http://dx.doi.org/10.1085/jgp.8.6.519

[10] Iqbal, M.A., Gupta, V., Gopinath, P., Mazurek, S. and Bamezai, R.N.K. (2014) Pyruvate Kinase M2 and Cancer: An Updated Assessment. FEBS Letters, 588, 2685-2692. http://dx.doi.org/10.1016/j.febslet.2014.04.011

[11] Pande, S.V. and Parvin, R. (1978) Pyruvate and Acetoacetate Transport in Mitochondria. A Reappraisal. Journal of Biological Chemistry, 253, 1565-1573.

[12] Shearman, M.S. and Halestrap, A.P. (1984) The Concentration of the Mitochondrial Pyruvate Carrier in Rat Liver and Heart Mitochondria Determined with Alpha-Cyano-Beta-(1-phenylindol-3-yl)acrylate. Biochemical Journal, 223, 673676.

[13] Bricker, D.K., Taylor, E.B., Schell, J.C., Orsak, T., Boutron, A., Chen, Y.C., Cox, J.E., Cardon, C.M., Van Vranken, J.G., Dephoure, N., Redin, C., Boudina, S., Gygi, S.P., Brivet, M., Thummel, C.S. and Rutter, J. (2012) A Mitochondrial Pyruvate Carrier Required for Pyruvate Uptake in Yeast, Drosophila, and Humans. Science, 337, 96-100. http://dx.doi.org/10.1126/science.1218099

[14] Herzig, S., Raemy, E., Montessuit, S., Veuthey, J.L., Zamboni, N., Westermann, B., Kunji, E.R.S. and Martinou, J.C. (2012) Identification and Functional Expression of the Mitochondrial Pyruvate Carrier. Science, 337, 93-96. http://dx.doi.org/10.1126/science.1218530

[15] Brailsford, M.A., Thompson, A.G., Kaderbhai, N. and Beechey, R.B. (1986) The Extraction and Reconstitution of the Alpha-Cyanocinnamate-Sensitive Pyruvate Transporter from Castor Bean Mitochondria. Biochemical and Biophysical Research Communications, 140, 1036-1042. http://dx.doi.org/10.1016/0006-291X(86)90739-4

[16] Nałecz, M.J., Nałecz, K.A., Broger, C., Bolli, R., Wojtczak, L. and Azzi, A. (1986) Extraction, Partial Purification and Functional Reconstitution of Two Mitochondrial Carriers Transporting Keto Acids: 2-Oxoglutarate and Pyruvate. FEBS Letters, 196, 331-336. http://dx.doi.org/10.1016/0014-5793(86)80273-3

[17] Capuano, F., Di Paola, M., Azzi, A. and Papa, S. (1990) The Monocarboxylate Carrier from Rat Liver Mitochondria. Purification and Kinetic Characterization in a Reconstituted System. FEBS Letters, 261, 39-42. http://dx.doi.org/10.1016/0014-5793(90)80631-R

[18] Nałecz, M.J., Nałecz, K.A. and Azzi, A. (1991) Purification and Functional Characterisation of the Pyruvate (Monocarboxylate) Carrier from Baker's Yeast Mitochondria (Saccharomyces cerevisiae). Biochimica et Biophysica Acta, 1079, 87-95. http://dx.doi.org/10.1016/0167-4838(91)90028-X

[19] Hildyard, J.C.W. and Halestrap, A.P. (2003) Identification of the Mitochondrial Pyruvate Carrier in Saccharomyces cerevisiae. Biochemical Journal, 374, 607-611. http://dx.doi.org/10.1042/BJ20030995

[20] Todisco, S., Agrimi, G., Castegna, A. and Palmieri, F. (2006) Identification of the Mitochondrial NAD ${ }^{+}$Transporter in Saccharomyces cerevisiae. Journal of Biological Chemistry, 281, 1524-1531. http://dx.doi.org/10.1074/jbc.M510425200

[21] Da Cruz, S., Xenarios, I., Langridge, J., Vilbois, F., Parone, P.A. and Martinou, J.C. (2003) Proteomic Analysis of the Mouse Liver Mitochondrial Inner Membrane. Journal of Biological Chemistry, 278, 41566-41571. http://dx.doi.org/10.1074/jbc.M304940200

[22] Halestrap, A.P. (2012) The Mitochondrial Pyruvate Carrier: Has It Been Unearthed at Last? Cell Metabolism, 16, 141-143. http://dx.doi.org/10.1016/j.cmet.2012.07.013

[23] Li, C.L., Wang, M., Ma, X.Y. and Zhang, W. (2014) NRGA1, a Putative Mitochondrial Pyruvate Carrier, Mediates ABA Regulation of Guard Cell Ion Channels and Drought Stress Responses in Arabidopsis. Molecular Plant, 7, 15081521. http://dx.doi.org/10.1093/mp/ssu061

[24] Lehmann, J.M., Moore, L.B., Smith-Oliver, T.A., Wilkison, W.O., Willson, T.M. and Kliewer, S.A. (1995) An Antidiabetic Thiazolidinedione Is a High Affinity Ligand for Peroxisome Proliferator-Activated Receptor $\gamma($ PPAR $\gamma$ ). Journal of Biological Chemistry, 270, 12953-12956. http://dx.doi.org/10.1074/jbc.270.22.12953

[25] Divakaruni, A.S., Wiley, S.E., Rogers, G.W., Andreyev, A.Y., Petrosyan, S., Loviscach, M., Wall, E.A., Yadava, N., Heuck, A.P., Ferrick, D.A., Henry, R.R., McDonald, W.G., Colca, J.R., Simon, M.I., Ciaraldi, T.P. and Murphy, A.N. (2013) Thiazolidinediones Are Acute, Specific Inhibitors of the Mitochondrial Pyruvate Carrier. Proceedings of the National Academy of Sciences of the United States of America, 110, 5422-5427.

http://dx.doi.org/10.1073/pnas.1303360110 
[26] Hardie, D.G. (2014) AMPK: Positive and Negative Regulation, and Its Role in Whole-Body Energy Homeostasis. Current Opinion in Cell Biology, 33C, 1-7.

[27] Colca, J.R., McDonald, W.G., Cavey, G.S., Cole, S.L., Holewa, D.D., Brightwell-Conrad, A.S., Wolfe, C.L., Wheeler, J.S., Coulter, K.R., Kilkuskie, P.M., Gracheva, E., Korshunova, Y., Trusgnich, M., Karr, R., Wiley, S.E., Divakaruni, A.S., Murphy, A.N., Vigueira, P.A., Finck, B.N. and Kletzien, R.F. (2013) Identification of a Mitochondrial Target of Thiazolidinedione Insulin Sensitizers (mTOT)—Relationship to Newly Identified Mitochondrial Pyruvate Carrier Proteins. PLoS ONE, 8, e61551. http://dx.doi.org/10.1371/journal.pone.0061551

[28] Vigueira, P.A., McCommis, K.S., Schweitzer, G.G., Remedi, M.S., Chambers, K.T., Fu, X., McDonald, W.G., Cole, S.L., Colca, J.R., Kletzien, R.F., Burgess, S.C. and Finck, B.N. (2014) Mitochondrial Pyruvate Carrier 2 Hypomorphism in Mice Leads to Defects in Glucose-Stimulated Insulin Secretion. Cell Reports, 7, 2042-2053. http://dx.doi.org/10.1016/j.celrep.2014.05.017

[29] Vacanti, N.M., Divakaruni, A.S., Green, C.R., Parker, S.J., Henry, R.R., Ciaraldi, T.P., Murphy, A.N. and Metallo, C.M. (2014) Regulation of Substrate Utilization by the Mitochondrial Pyruvate Carrier. Molecular Cell, 56, 425-435. http://dx.doi.org/10.1016/j.molcel.2014.09.024

[30] Yang, C., Sudderth, J., Dang, T., Bachoo, R.M., Bachoo, R.G., McDonald, J.G. and DeBerardinis, R.J. (2009) Glioblastoma Cells Require Glutamate Dehydrogenase to Survive Impairments of Glucose Metabolism or Akt Signaling. Cancer Research, 69, 7986-7993. http://dx.doi.org/10.1158/0008-5472.CAN-09-2266

[31] Yang, C., Ko, B., Hensley, C.T., Jiang, L., Wasti, A.T., Kim, J., Sudderth, J., Calvaruso, M.A., Lumata, L., Mitsche, M., Rutter, J., Merritt, M.E. and DeBerardinis, R.J. (2014) Glutamine Oxidation Maintains the TCA Cycle and Cell Survival during Impaired Mitochondrial Pyruvate Transport. Molecular Cell, 56, 414-424. http://dx.doi.org/10.1016/j.molcel.2014.09.025

[32] Cairns, R.A., Harris, I.S. and Mak, T.W. (2011) Regulation of Cancer Cell Metabolism. Nature Reviews Cancer, 11, 85-95. http://dx.doi.org/10.1038/nrc2981

[33] Schell, J.C., Olson, K.A., Jiang, L., Hawkins, A.J., Van Vranken, J.G., Xie, J., Egnatchik, R.A., Earl, E.G., DeBerardinis, R.J. and Rutter, J. (2014) A Role for the Mitochondrial Pyruvate Carrier as a Repressor of the Warburg Effect and Colon Cancer Cell Growth. Molecular Cell, 56, 400-413. http://dx.doi.org/10.1016/j.molcel.2014.09.026

[34] Moreb, J.S. (2008) Aldehyde Dehydrogenase as a Marker for Stem Cells. Current Stem Cell Research \& Therapy, 3 , 237-246. http://dx.doi.org/10.2174/157488808786734006

[35] Tirino, V., Desiderio, V., Paino, F., De Rosa, A., Papaccio, F., La Noce, M., Laino, L., De Francesco, F. and Papaccio, G. (2013) Cancer Stem Cells in Solid Tumors: An Overview and New Approaches for Their Isolation and Characterization. FASEB Journal, 27, 13-24. http://dx.doi.org/10.1096/fj.12-218222

[36] Leushacke, M. and Barker, N. (2012) Lgr5 and Lgr6 as Markers to Study Adult Stem Cell Roles in Self-Renewal and Cancer. Oncogene, 31, 3009-3022. http://dx.doi.org/10.1038/onc.2011.479

[37] Zhou, J., Ng, S.B. and Chng, W.J. (2013) LIN28/LIN28B: An Emerging Oncogenic Driver in Cancer Stem Cells. International Journal of Biochemistry \& Cell Biology, 45, 973-978. http://dx.doi.org/10.1016/j.biocel.2013.02.006

[38] Iv Santaliz-Ruiz, L.E., Xie, X., Old, M., Teknos, T.N. and Pan, Q. (2014) Emerging Role of Nanog in Tumorigenesis and Cancer Stem Cells. International Journal of Cancer, 135, 2741-2748. http://dx.doi.org/10.1002/ijc.28690 
Scientific Research Publishing (SCIRP) is one of the largest Open Access journal publishers. It is currently publishing more than 200 open access, online, peer-reviewed journals covering a wide range of academic disciplines. SCIRP serves the worldwide academic communities and contributes to the progress and application of science with its publication.

Other selected journals from SCIRP are listed as below. Submit your manuscript to us via either submit@scirp.org or Online Submission Portal.
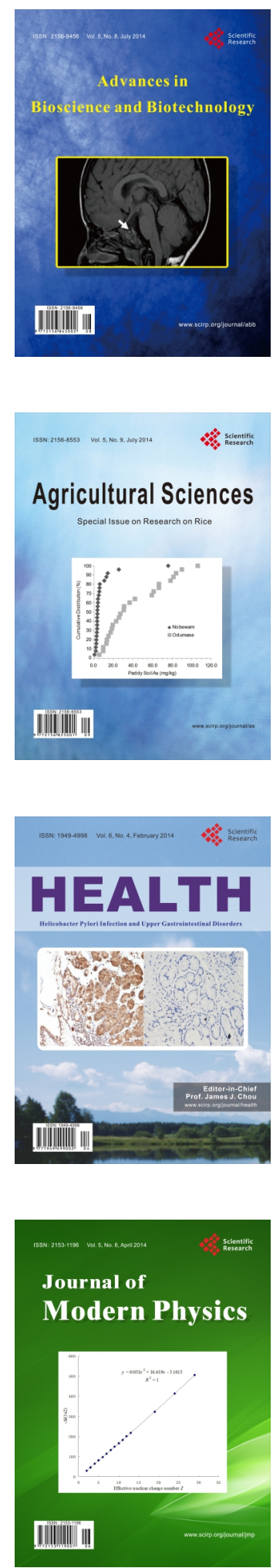
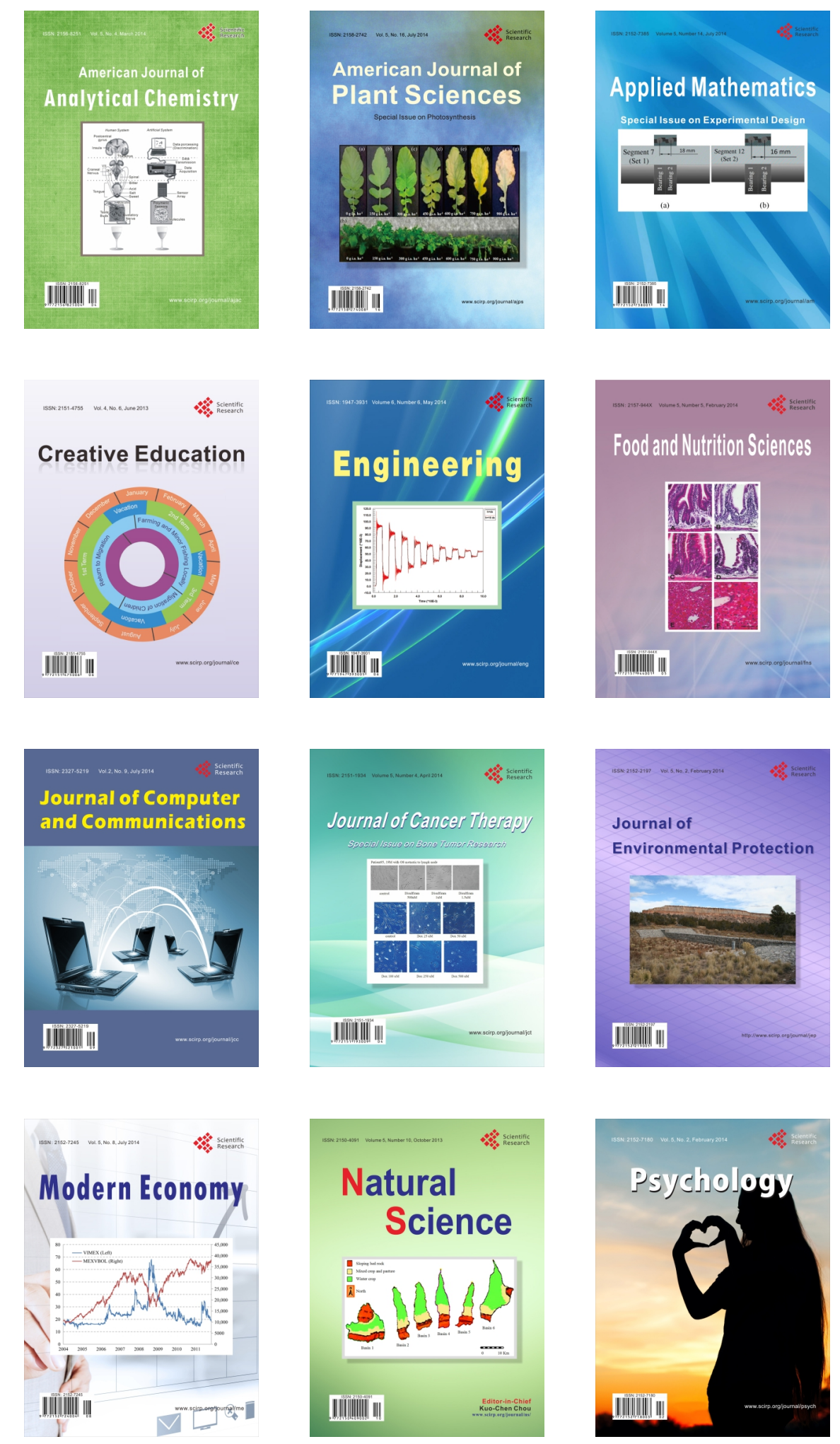